\title{
SOME ASYMPTOTIC FORMULAS FOR MULTIPLICATIVE FUNCTIONS
}

\author{
P. ERDÖS
}

The present paper contains several asymptotic formulas for the sum of multiplicative functions. A function $f(n)$ is called multiplicative if $f(a \cdot b)=f(a) \cdot f(b)$ for $(a, b)=1$. We assume $f(n)>0$. In this paper $f(n), f_{1}(n)$ will always denote multiplicative functions. First we prove the following theorem.

Theorem $1 .{ }^{1}$ Assume that the two series

$$
-\sum_{p, \alpha} \frac{f\left(p^{\alpha}\right)-1}{p^{\alpha}}, \quad \sum_{p, \alpha} \frac{\left(f\left(p^{\alpha}\right)-1\right)^{2}}{p^{\alpha}},
$$

converge; then $f(n)$ has a mean value, that is,

$$
\lim _{n \rightarrow \infty} \frac{1}{n} \sum f(m)
$$

exists and is not equal to zero.

This result was conjectured in a slightly more special form at the end of my paper Some remarks on additive and multiplicative functions. ${ }^{2}$

REMARK. The convergence of (1) is the necessary and sufficient condition for the existence of the distribution function of $f(n) .^{3}$

For the sake of simplicity we assume $f\left(p^{\alpha}\right)=f(p)$. Then we prove

$$
\lim _{n \rightarrow \infty} \frac{1}{n} \sum_{m \leqq n} f(m)=\prod_{p}\left(1+\frac{f(p)-1}{p}\right) .
$$

It easily follows from (1) that the product on the right side of (2) converges and thus the value of the limit is not 0 .

We easily obtain from (1) that for every $\epsilon>0$

$$
\sum_{|f(p)-1|>e} \frac{1}{p}<\infty \text {. }
$$

Received by the editors May 27, 1946, and, in revised form, December 11, 1946. $481-485$.

${ }^{1}$ This result generalizes a result of Wintner, Amer. J. Math. vol. 67 (1945) pp.

2 Bull. Amer. Math. Soc. vol. 52 (1946) pp. 527-537.

${ }^{3}$ P. Erdös and A. Wintner, Amer. J. Math. vol. 61 (1939) pp. 713-721. See also footnote 2 . 
Thus by arguments used in previous papers ${ }^{4}$ we can assume, for the sake of simplicity (without loss of generality), that $f(p) \rightarrow 1$ as $p \rightarrow \infty$.

Define

$$
f_{k}(m)=\prod_{p \mid m, p \leq p_{k}} f(p) .
$$

Also $M(x, n)$ and $M_{k}(x, n)$ (x large) denote the number of integers $m \leqq n$ for which $f(m) \geqq x, f_{k}(m) \geqq x$, respectively. Further let

$$
A(m)=\prod_{p^{\alpha} \| \mid m}^{\prime} p^{\alpha}, \quad B(m)=\prod_{p^{\alpha} \| m}^{\prime \prime} p^{\alpha} \quad(m=A(m) \cdot B(m)) .
$$

Here $p^{\alpha} \| m$ means that $p^{\alpha} \mid m$ and $p^{\alpha+1}\{m$, and the prime denotes that the product is extended over the $p \leqq n^{1 / x^{10}}$, and the double prime denotes that the product is extended over the $p>n^{1 / x^{10}}$.

First we have to prove some lemmas.

LEMMA 1. The number $N$ of integers $m \leqq n$ with

$$
A(m) \geqq n^{1 / 2}
$$

is $o\left(n / x^{4}\right)$ (we assume that $x \rightarrow \infty$ ).

In the product $\prod_{m \leqq n} A(m)$, the prime $p$ occurs as many times as $p$ divides $n$ (and $p \leqq n^{1 / x^{10}}$ ). Hence,

$$
\prod_{m \leqq n} A(m)<\prod_{p}^{\prime} p^{n /(p-1)}<\exp \left(\frac{c n \log n}{x^{10}}\right)
$$

since $\sum_{p \leqq x} \log p / p<c \log x$ (the prime means $p \leqq n^{1 / x^{10}}$ ). Multiplying together the inequalities $n^{1 / 2} \leqq A(m)$ we have

$$
n^{N / 2} \leqq \prod_{m \leqq n} A(m)<\exp \left(\frac{c n \log n}{x^{10}}\right)
$$

or

$$
N<c \frac{2 n}{x^{10}}
$$

which proves the lemma.

LEMmA 2. The number $N^{\prime}$ of integers $m \leqq n$ with $f(B(m)) \geqq x^{1 / 2}$ is $o\left(n / x^{4}\right)$.

4 P. Erdös, J. London Math. Soc. vol. 10 (1935) p. 124. (By the method used there we can show that without loss of generality we can neglect a sequence of primes $p_{i}$ with $\sum 1 / p_{i}<\infty$.) 
Since $f(p) \rightarrow 1$ and all prime factors of $B(m)$ are greater than $n^{1 / x^{10}}$, we obtain that if $p \mid B(m), f(p)<1+\epsilon$. Thus if $f(B(m)) \geqq x^{1 / 2}$ we conclude that $m$ has at least $100 \log x$ prime factors greater than $n^{1 / x^{10}}$. Thus on the one hand

$$
\sum_{f(B(m)) \geqq x^{1 / 2}}^{*} \frac{n}{B(m)}>N^{\prime}
$$

on the other hand, where the star indicates that each $B(m)$ is counted only once,

$$
\sum_{f(B(m)) \geqq x^{1 / 2}}^{*} \frac{1}{B(m)}<\left(\sum^{\prime} \frac{1}{p}\right)^{u} / u !,
$$

where $u=[100 \log x]$ and the prime indicates that $n^{1 / x^{10}}<p \leqq n$. Now

$$
\sum^{\prime} \frac{1}{p}<\log \log n-\log \log \left(n^{1 / x^{10}}\right)+o(1)<11 \log x
$$

hence

$$
N^{\prime}<n \frac{(11 \log x)^{u}}{u !}=o\left(\frac{n}{x^{4}}\right),
$$

which proves the lemma.

LEMMA 3. There exists an absolute constant c (independent of $k$ ) such that for all $x$ and $n$

$$
M(x, n)<c n / x^{3} \quad \text { and } M_{k}(x, n)<c n / x^{3} .
$$

REMARK. Lemma 3 is not trivial only for large $x$ and $n$. It will be clear from the proof that the lemma is true with an arbitrary $t$ instead of 3 . It will be clear from the proof that it suffices to consider $M(x, n)$.

Suppose the lemma is false. Then we clearly can assume that there exist infinite sequences $x_{i}$ and $n_{i}$ such that

$$
M\left(x_{i}, n_{i}\right)>1 / x_{i}^{4}, \quad x_{i} \rightarrow \infty, n_{i} \rightarrow \infty .
$$

Let $a_{1}<a_{2}<\cdots<a_{k} \leqq n_{i}$ be the integers not greater than $n_{i}$ with $f\left(a_{j}\right) \geqq x$. For simplicity of notation we replace $x_{i}$ by $x$ and $n_{i}$ by $n$ where there is no danger of confusion. We obtain from Lemmas 1 and 2 that there exist at least $n / 2 x^{4} a$ 's for which

$$
f\left(A\left(a_{j}\right)\right)>x^{1 / 2}, \quad A\left(a_{j}\right)<n^{1 / 2} \quad(\text { since } f(m)=f(A(m)) f(B(m))) .
$$

Denote these $a$ 's by $a^{+}$. Consider the integers $m \leqq n$ for which $A(m)$ $=t$. We must have $m=t v \leqq n$, where $v$ is not divisible by any $p \leqq n^{1 / x^{10}}$, 
since these primes are included in $t$, and $v \leqq n / t$. The number of such integers $v$ is equal to the number of integers $m \leqq n$ with $A(m)=t$. Since we are later going to let $t$ run through the $A\left(a_{j}^{+}\right)$, it is permissible to assume $t<n^{1 / 2}$ by (4). Then Brun's method ${ }^{5}$ yields the result

$$
\sum_{A(m)=t} 1 \leqq c \frac{n}{t} \prod_{p}^{\prime}\left(1-\frac{1}{p}\right)<c \frac{n x^{10}}{t \log n} ;
$$

the prime indicates that $p \leqq n^{1 / x^{10}}$. Now letting $t$ run through the $A\left(a_{j}^{+}\right)$we obtain that the number of $a_{j}^{+}$'s satisfying (4) is less than

$$
\sum^{\prime}\left(c / A\left(a_{i}^{+}\right)\right)\left(n x^{10} / \log n\right)
$$

(the prime indicates that each $A\left(a_{j}^{+}\right)$is counted only once); on the other hand the number of $a_{j}^{+}$'s is equal to or greater than $n / 2 x^{4}$ as stated in the lines preceding (4). Hence

$$
\sum^{\prime} \frac{c}{A\left(a_{j}^{+}\right)} \frac{n x^{10}}{\log n}>\frac{1}{2} \frac{n}{x^{4}} .
$$

Let now $N$ tend to infinity. As above we have that the number of integers $m \leqq N$ for which $A(m)=t$ equals

$$
(1+o(1)) \frac{N}{t} \prod_{p}^{\prime}\left(1-\frac{1}{p}\right)>\frac{c N}{t} \frac{x^{10}}{\log n},
$$

with a new value for the constant $c$. Thus we obtain from (7) that the number of integers $m \leqq N$ with $A(m)=A\left(a_{j}^{+}\right), j=1,2, \cdots$, is greater than $c N / x^{4}$. But for these integers we have by (4)

$$
f_{k}(m)=f(A(m)) \geqq x^{1 / 2} \quad\left(k=\pi\left(n^{1 / x^{10}}\right)\right) .
$$

Thus for all sufficiently large $N$

$$
M_{k}\left(x_{i}^{1 / 2}, N\right)>c N / x_{i}^{4} \quad\left(x_{i}=x\right)
$$

where $c$ is an absolute constant and $x_{i}$ and $k$ are independent of $N$. From (9) we obtain by a simple calculation that

$$
\sum_{m=1}^{N}\left(f_{k}(m)\right)^{10}>c x_{i} N
$$

and $x_{i}$ can assume arbitrarily large values. Now we shall show that (10) is false; in fact we prove the following lemma.

$5 \mathrm{~V}$. Brun, Le crible d'Eratosthene et le théorème de Goldbach, Skrifter udgivne af Videnskabs selskabet Kristiania, Si Matematisk-Naturvidenskapelig Klasse vol. 3 (1920). 
LEMma 4. Put $h_{t}(p)=(f(p))^{t}-1$. We have

$$
\sum_{m=1}^{N} f_{k}(m)^{t}=(1+o(1)) N \prod_{p \leqq p_{k}}\left(1+\frac{h_{t}(p)}{p}\right)<c N,
$$

where $c=c(t)$ is independent of $k$.

We have

$$
\begin{aligned}
\sum_{m=1}^{N} f_{k}(m)^{t} & =\sum_{m=1}^{N} \prod_{p \mid m, p \geqq p_{k}}\left(1+h_{t}(p)\right)=\sum_{d}^{\prime}\left[\frac{N}{d}\right] \prod_{p \mid d} h_{t}(p) \\
& =N \sum_{d} \frac{\prod_{p \mid d} h_{t}(p)}{d}+O(1)=(1+o(1)) N \prod_{p \leqq p_{k}}\left(1+\frac{h_{t}(p)}{p}\right),
\end{aligned}
$$

where the dash indicates that $d$ is squarefree and that all prime factors of $d$ are not greater than $p_{k}$, and the error term $O(1)$ depends on $k$ but not on $N$ (the number of terms in $\sum^{\prime}$ is bounded, and the bound depends on $k$ but not on $N$ ). The second inequality of (11) follows easily from (1). This proves Lemma 4, and since (11) contradicts (10), Lemma 3 is also proved.

Now we can prove Theorem 1 . For $t=1$ we obtain from (11)

$$
\lim _{n \rightarrow \infty} \frac{1}{n} \sum_{m=1}^{n} f_{k}(m)=\operatorname{II}_{p \leqq p_{k}}\left(1+\frac{f(p)-1}{p}\right) .
$$

Further from (1) we have

$$
\lim _{k \rightarrow \infty} \prod_{p \leqq p_{k}}\left(1+\frac{f(p)-1}{p}\right)=\prod_{p=2}^{\infty}\left(1+\frac{f(p)-1}{p}\right) .
$$

Thus to prove (2) (that is, Theorem 1) it will suffice to show that for every $\epsilon$ there exists a $k_{0}$ so that for $k>k_{0}$ and all sufficiently large $n$

$$
\frac{1}{n}\left|\sum_{m=1}^{n} f(m)-f_{k}(m)\right|<\epsilon \text {. }
$$

Write

$$
\sum_{m=1}^{n}\left(f(m)-f_{l}(m)\right)=\sum_{1}+\sum_{2},
$$

where in $\sum_{1}$ the summation is extended over the $m \leqq n$ for which $f(m) \leqq t$ and $f_{k}(m) \leqq t\left(t\right.$ fixed), and in $\sum_{2}$ the summation is extended over the remaining $m \leqq n$.

Consider $\sum_{2}$. Divide the integers $m$ appropriate to $\sum_{2}$ into classes 
$m_{i}(i=t, t+1, \cdots)$ such that $i \leqq f\left(m_{i}\right)<i+1$. Then applying Lemma 3 , we get

$$
\begin{aligned}
\sum_{2} & \leqq \sum_{f(m) \geqq t} f(m)+\sum_{f_{k}(m) \geqq t} f_{k}(m)=\sum_{i=t}^{\infty} f\left(m_{i}\right)+\sum_{k=t}^{\infty} f_{k}\left(m_{i}\right) \\
& <2 c n \sum_{i=t}^{\infty} \frac{i+1}{i^{3}}<\frac{\epsilon}{2} n,
\end{aligned}
$$

for $t$ sufficiently large. We now fix $t_{0}$ so that for all $t>t_{0}$, the last inequality of (16) holds.

Now we estimate $\sum_{1}$. Let

$$
\sum_{1}=\sum_{1}^{\prime}+\sum_{1}^{\prime}
$$

where in $\sum_{1}^{\prime}$ we impose the condition $\left|f(m)-f_{k}(m)\right|>\epsilon / 4$, and in $\sum_{1}^{\prime \prime}$ we require $\left|f(m)-f_{k}(m)\right| \leqq \epsilon / 4$. Then in $\sum_{1}^{\prime \prime}$ there are at most $n$ summands each numerically less than $\epsilon / 4$, so this sum is numerically less than $\epsilon n / 4$. In $\sum_{1}^{\prime}$ each summand exceeds $\epsilon / 4$ in absolute value but is less than $t$ by our definition of $\sum_{1}$. Moreover by taking $k$ sufficiently large we can insure that the number of summands in $\sum_{1}$ ' will be less than $\epsilon n / 4 t$. For it is well known that both $f_{k}(m)$ and $f(m)$ have asymptotic distribution functions and that the asymptotic distribution function of $f_{k}(m)$ tends to that of $f(m)$ as $k \rightarrow \infty . .^{6}$ But this implies that $f_{k}(n)$ tends to $f(n)$ in relative measure, that is, that the number of integers not exceeding $n$ for which $\left|f(n)-f_{k}(n)\right|>\epsilon$ tends to zero with $1 / k$. Hence, we have the estimate

$$
\left|\sum_{1}\right|=\left|\sum_{1}\right|+\left|\sum_{1}^{\prime}\right|<\frac{\epsilon n}{4 t} \cdot t+\frac{\epsilon n}{4}<\frac{\epsilon n}{2}
$$

and

$$
\left|\sum_{m=1}^{n}\left(f(m)-f_{k}(m)\right)\right|=\left|\sum_{1}\right|+\left|\sum_{2}\right|<\epsilon n .
$$

This completes the proof.

It seems possible that

$$
\lim _{n \rightarrow \infty} \frac{1}{n} \sum_{m=1}^{n} f(m)
$$

exists if we only assume that $\sum f(p) / p$ converges (some assumption like $|f(p)|<c$ might also be necessary). At present I am unable to decide this question. The present proof breaks down since Lemma 4

${ }^{6}$ Ibid. footnote 3. 
is false if $\sum f(p) / p$ converges and $\sum(f(p))^{2} / p$ diverges.

Ramanujan ${ }^{7}$ conjectured that for any $\alpha>0, \beta>0$

$$
\begin{aligned}
& \sum_{\nu=1}^{n} \sigma_{\alpha}(\nu) \sigma_{\beta}(n-\nu) \\
& =(1+o(1)) \frac{\Gamma(\alpha+1) \Gamma(\beta+1)}{\Gamma(\alpha+\beta+2)} \frac{\zeta(\alpha+1) \zeta(\beta+1)}{\zeta(\alpha+\beta+2)} \sigma_{\alpha+\beta+1}(n),
\end{aligned}
$$

where $\sigma_{\alpha}(n)$ denotes the sum of the $\alpha$ th power of the divisors of $n$.

Ingham ${ }^{8}$ proved this conjecture; he also found analogous asymptotic formulas for $\sum_{\nu=1}^{n} \sigma_{\alpha}(\nu) \sigma_{\alpha}(\nu+k), \sum_{\nu=1}^{n} \phi(\nu) \phi(\nu+k), \sum_{\nu=1}^{n} d(\nu) d(n-\nu)$, $\sum_{\nu=1}^{n} d(\nu) d(\nu+k)$.

We are going to generalize these results. First we prove the following theorem.

THEOREM 2. Assume that $f^{(1)}(m)$ and $f^{(2)}(m)$ satisfy (1), also $f^{(i)}\left(p^{\alpha}\right)=f^{(i)}(p), i=1,2$. Put $f^{(i)}(p)-1=g^{(i)}(p), i=1$, 2. Then

$$
\begin{aligned}
& \sum_{\nu=1}^{n} f^{(1)}(\nu) f^{(2)}(n-\nu)=(1+o(1)) A n, \\
& \sum_{\nu=1}^{x} f^{(1)}(\nu) f^{(2)}(\nu+n)=(1+o(1)) A x,
\end{aligned}
$$

where

$$
\begin{aligned}
A= & \prod_{p X_{n}}\left(1+\frac{g^{(1)}(p)+g^{(2)}(p)}{p}\right) \\
& \cdot \prod_{p \mid n}\left(1+\frac{g^{(1)}(p)+g^{(2)}(p)+g^{(1)}(p) g^{(2)}(p)}{p}\right) .
\end{aligned}
$$

Remarks. (1) It clearly follows from (1) that the product for $A$ converges. (2) The assumption $f\left(p^{\alpha}\right)=f(p)$ can be omitted without any difficulty but the expression for $A$ then becomes much more complicated.

We have by a simple calculation $\left(g_{k}(p)=f_{k}(p)-1\right)$

$$
\begin{aligned}
\sum_{\nu=1}^{n} f_{k}^{(1)}(\nu) f_{k}^{(2)}(n-\nu) & =\sum_{\nu=1}^{n}\left(\prod_{p \mid \nu}\left(1+g_{k}^{(1)}(p)\right) \prod_{p \mid n-\nu} 1+g_{k}^{(2)}(n-\nu)\right) \\
& =\sum_{d_{1}, d_{2} \geq_{1}}\left[\frac{n}{d_{1} d_{2}}\right]^{\prime} \prod_{p \mid d_{1}} g_{k}^{(1)}(p) \prod_{p \mid d_{2}} g_{k}^{(2)}(p),
\end{aligned}
$$

7 Collected papers, p. 137.

8 J. London Math. Soc. vol. 2 (1927) pp. 202-208. 
where $\left[n / d_{1} d_{2}\right]$ denotes the number of solutions of $n=d_{1} x+d_{2} y$ in positive integers $x$ and $y$. Clearly $\left|n / d_{1} d_{2}-\left[n / d_{1} d_{2}\right]^{\prime}\right| \leqq 1$. Thus a simple calculation shows that

$$
\begin{aligned}
\sum_{p=1}^{n} f_{k}^{(1)}(\nu) f_{k}^{(2)}(n-\nu)= & (1+o(1)) n \prod_{p+m, p \leqq p_{k}}\left(\frac{1+g^{(1)}(p)+g^{(2)}(p)}{p}\right) \\
& \cdot \prod_{p \mid n, p \leqq p_{k}}\left(1+\frac{g^{(1)}(p)+g^{(2)}(p)+g^{(1)}(p) g^{(2)}(p)}{p}\right) .
\end{aligned}
$$

Since the product for $A$ converges our proof will be complete if we show that for sufficiently large $k$

$$
\left|\sum_{\nu=1}^{n}\left\{f^{(1)}(\nu) f^{(2)}(n-\nu)-f_{k}^{(1)}(\nu) f_{k}^{(2)}(n-\nu)\right\}\right|<\epsilon n .
$$

We suppress the proof of (18) since it is almost identical with that of (14); this completes the proof of the first half of Theorem 2. The proof of the second half is similar.

By the same method we can prove

$$
\begin{aligned}
& \sum F_{k}^{(1)}(\nu) F_{k}^{(2)}(n-\nu)=(1+o(1)) A \frac{\Gamma(\alpha+1) \Gamma(\beta+1)}{\Gamma(\alpha+\beta+2)} n^{\alpha+\beta+1}, \\
& \sum_{\nu=1}^{x} F^{(1)}(\nu) F^{(2)}(\nu+n)=(1+o(1)) \frac{A}{\alpha+\beta+1} x^{\alpha+\beta+1} ;
\end{aligned}
$$

where $F^{(1)}(n)=n^{\alpha} f^{(1)}(n), F^{(2)}(n)=n^{\beta} f^{(2)}(n), \alpha>0, \beta>0$ are arbitrary real numbers. (19) contains all the results of Ingham except those on $d(n)$ (we of course have to drop the assumption $f\left(p^{\alpha}\right)=f(p)$ ).

Carlitz proved the following theorem: ${ }^{9}$ Let

$$
f(p)=1+o\left(p^{-1 / 2+o}\right) \text {. }
$$

Then

$$
\sum^{\prime} f\left(n_{1}\right) f\left(n_{2}\right) \cdots f\left(n_{\nu}\right)=C n^{\nu-1}+O\left(n^{\nu-9 / 8+e}\right) ;
$$

the prime means that the summation is extended over all partitions of $n$ into $v$ summands. The value of $C$ is given by a complicated expression.

By the method we used in proving Theorem 2 we can prove the following theorem.

THEOREM 3. Let $f^{i}(n)$ satisfy (1), $i=1,2, \cdots, \nu$. Then

${ }^{9}$ Quart. J. Math. Oxford Ser. vol. 2 (1931) pp. 97-106; see also vol. 3 (1932) pp. 273-290. 


$$
\sum^{\prime} f^{(1)}\left(n_{1}\right) f^{(2)}\left(n_{2}\right) \cdots f^{(\nu)}\left(n_{\nu}\right)=(1+o(1)) D n^{\nu-1},
$$

also

$$
\sum_{m=1}^{n} f^{(1)}\left(m+k_{1}\right) f^{(2)}\left(m+k_{2}\right) \cdots f^{(v)}\left(m+k_{\nu}\right)=(1+o(1)) E n,
$$

$D$ and $E$ are given by a complicated expression.

STANFORD UNIVERSITY

\section{ON A CLASS OF TAYLOR SERIES}

\section{F. COWLING}

1. Introduction. Consider the Taylor series $\sum_{n=0}^{\infty} a_{n} z^{n}$. Suppose that the singularities of the function defined by the series all lie in certain regions of the complex plane and that the coefficients possess certain arithmetical properties. Mandelbrojt ${ }^{1}$ has shown that under restrictions of this nature it is possible to predict the form of the function defined by the series. This note is concerned with the establishing of a new method to obtain more general results of this nature.

2. The method. The method that is employed here is an adaptation of a method used by Lindelöf [2] in the problem of representation of a function defined by a series.

Let $f(z)$ be regular in a region $D$ of the complex plane. Suppose that there exists a linear transformation $t=h(z)$ which maps the region of regularity into a region which includes the unit circle of the $t$-plane in its interior. Let $z=g(t)$ be the inverse of this transformation. Then $F(t)=f(g(t))$ is regular in this region in the $t$-plane. For this note it is convenient to suppose that $z=0$ corresponds to $t=0$ in the mapping. We may expand $g(t)$ in a Taylor series about $t=0$ and obtain

$$
z=b_{1} t+b_{2} t^{2}+\cdots
$$

convergent for $t$ in absolute value sufficiently small. Let

$$
f(z)=\sum_{n=0}^{\infty} a_{n} z^{n}
$$

Received by the editors August 2, 1946.

${ }^{1}$ See, Mandelbrojt [3]. Numbers in brackets refer to the bibliography at the end of the paper. 